\title{
ISOLAMENTO VISUAL OU ESPACIAL EM RUMINANTES
}

\author{
VISUAL OR SPATIAL ISOLATION IN RUMINANTS \\ Santana Júnior, H.A. ${ }^{1 \mathrm{~A}}$, Cardoso, E.O. ${ }^{1 \mathrm{~B}}$, Silva, F.F. ${ }^{1 \mathrm{C}}$, Marques, J.A. ${ }^{2}$, Mendes, F.B.L. ${ }^{1 \mathrm{D}}$ \\ e Abreu Filho, G. ${ }^{1 \mathrm{G}}$
}

\begin{abstract}
${ }^{1}$ Universidade Estadual do Sudoeste da Bahia. Itapetinga. Bahia. Brasil. Ahsantanajunior@hotmail; Beocardoso@hotmail.com; ${ }^{\text {Cffsilva@pq.cnpq.br; Dfabricio.bacelar@hotmail.com; }{ }^{6} g e o r g e a b r e u 16 @ h o t m a i l . ~}$ com

${ }^{2}$ Universidade Federal do Recôncavo Baiano. Brasil. jairmarques@hotmail.com
\end{abstract}

PaLAVRAS ChaVE ADICIONAIS

Estresse. Etologia. Separação. Social.

\section{RESUMO}

A produção animal em sistemas intensivos tem se ampliado no mundo, sempre visando à elevação de índices produtivos e retorno econômico, respectivamente. Essa verticalização da pecuária tem provocado algumas adaptações de manejo e também nas instalações, que tem alterado o comportamento dos animais, oriundo das restrições tanto visual como espacial do indivíduo. Na pecuária leiteira, a criação de bezerros em baias individuais é um exemplo dessa verticalização, tornando então uma prática comum em muitos países. Sabendo que os bovinos são animais sociais que mostram alta disponibilidade para formar vínculo social com outros indivíduos da mesma espécie, alguns órgãos têm implantado legislações para garantir um mínimo de conforto para esses animais. Um estudo sistemático das preferências sociais dos animais e os efeitos calmantes do animal companheiro de familiaridade diferente são necessários para documentar a resposta à separação. Objetivou-se com esta revisão, apresentar e discutir o efeito do isolamento espacial e/ou visual sobre as respostas produtivas e comportamentais de animais ruminantes.

\section{SUMMARY}

Livestock production in intensive systems has expanded the world, always agreeing to raise production levels and economic returns, respectively. This vertical integration of livestock has led to some adjustments in management and
AdDITIONAL KEYWORDS

Stress. Ethology. Separation. Social. also on the premises, which has changed the behavior of animals, coming from both visual and spatial constraints of the individual. In dairy farming, the creation of calves in individual stalls is an example of vertical integration, thus making a common practice in many countries. Knowing that the cattle are social animals that show high willingness to form social bonds with other individuals of the same species, some agencies have implemented laws to ensure a minimum of comfort for these animals. A systematic study of social preferences of animals and the calming effects of different animal companion familiarity are needed to document the response to separation. The objective of this review, present and discuss the effect of spatial isolation and/or visual on the productive and behavioral responses of ruminant animals.

\section{INTRODUÇÃO}

A produção animal em sistemas intensivos tem se ampliado no mundo, sempre visando à elevação de índices produtivos e retorno econômico, respectivamente. Essa verticalização da pecuária tem provocado algumas adaptações de manejo e também nas instalações, que tem alterado o comportamento dos animais, oriundo das restrições tanto visual como espacial do indivíduo. 
Na pecuária leiteira, a criação de bezerros em baias individuais é um exemplo dessa verticalização, tornando então uma prática comum em muitos países (Fredriksson et al., 2006). Segundo Eriksson (2009), a utilização de baias individuais tem muitas vantagens, dentre elas, a mais pronunciada é a redução da transmissão de doenças entre os bezerros. Isto é explicado pelo fato de que bezerros em baias individuais têm o contato físico muito limitado com as outras baias. Além disso, as criações individuais são mais fáceis de controle sanitário, a fim de detectar os sinais de doença.

Portanto, sob outro âmbito de visão, verifica-se que a intensificação dos sistemas, acarreta alguns prejuízos ao animal, em consequência da alteração do seu comportamento normal. Seguindo o mesmo exemplo, as baias individuais para criação de bezerros possuem vários inconvenientes, o mais visível é a limitação física de espaço que oferece um número limitado ou ausente de oportunidade de contato social com outros bezerros, impossibilitando o desenvolvimento do seu comportamento normal (Faerevik et al., 2006).

Sabendo que os bovinos são animais sociais que mostram alta disponibilidade para formar vínculo social com outros indivíduos da mesma espécie (Esteves et al., 2007), alguns órgãos tem implantado legislações para garantir um mínimo de conforto para esses animais. A fim de satisfazer a necessidade de vitelos para o contato social, a habitação para grupos de bezerros com idade superior a oito semanas de idade passou a ser obrigatória na União Européia (EU Legislation Council Directive 97/2/EEC). Pois quando comparado à habitação em pequenas baias individuais, o alojamento em grupo dá aos bezerros uma maior oportunidade de realizar brincadeiras, no entanto, mais conhecimentos sobre as preferências sociais e as reações à separação dos animais se faz necessário a fim de compreender as consequências do bem- estar do reagrupamento e da mistura de indivíduos desconhecidos (Faerevik et al., 2006).

Na pecuária, grupos homogêneos de animais são formados de acordo com o objetivo de produção, condição corporal, peso, ingestão alimentar e do estado de saúde. Com isso, despreza-se a importância da manutenção do vínculo social estabelecido entre cada animal. Ao isolar animais, os mesmos apresentam maior número de vocalizações, com intensidades diferentes, bem como aumentam respostas fisiológicas de estresse, tais como frequência cardíaca e níveis plasmáticos de cortisol (Mckittrick et al., 2009).

A presença, ou a mera visão da mesma espécie promove alterações na reação comportamental. Faerevik et al. (2006) afirmam que a presença e a familiaridade de um companheiro possui efeito calmante de um animal de produção e deve ser observado durante o reagrupamento, transporte e movimentação. Um estudo sistemático das preferências sociais dos animais e os efeitos calmantes do animal companheiro de familiaridade diferente são necessários para documentar a resposta à separação.

Quando comparado aos seres humanos, os bovinos são animais grandes e fortes, com isso, técnicas específicas e instalações tiveram que ser criados com o objetivo de que os seres humanos pudessem lidar com eles de forma segura. No entanto, mesmo quando operam dentro dos limites das técnicas e das instalações, o manejo dos animais contém um elevado grau de perigo, portanto a magnitude da reação animal não parece ser constante entre os mesmos, devido a diferenças individuais no seu comportamento. Por esta razão, pesquisadores têm tentado desenvolver testes para prever o nível de perigo que o animal isolado individualmente possa provocar para os manipuladores (Kilgour et al., 2006).

Objetivou-se com esta revisão, apresentar e discutir o efeito do isolamento 
Tabela I. Descrição dos comportamentos registrados para bezerros. (Description of behavior recorded for calves).

\begin{tabular}{ll}
\hline Comportamento & Descrição \\
\hline Contato social estimulador & Nariz a menos de $10 \mathrm{~cm}$ de distância de qualquer parte do corpo do vitelo. \\
Imobilidade & Ficar parado por 5 segundos ou mais. \\
Exploração & Cheirar a menos de $10 \mathrm{~cm}$, ou lamber, as paredes ou o piso. \\
Locomoção & Número de passos (contados a partir da movimentação da pata dianteira \\
& direita). \\
\hline
\end{tabular}

Fonte: Faerevik et al. (2006).

espacial e/ou visual sobre as respostas produtivas e comportamentais de animais ruminantes.

\section{EFEITOS SOBRE OS RUMINANTES}

Kondo e Hurnik (1988) relataram que os animais colocados em um ambiente desconhecido reagem com medo, que é expresso como uma resposta ao estresse e caracterizado por elevada frequência cardíaca, aumento dos níveis de cortisol plasmático e os sinais de comportamento de agitação, tais como aumento da vocalização, micção e defecação. Animais em geral, também apresenta essa resposta quando visualmente isolados dos companheiros de rebanho (Piller et al., 1999).

A vocalização é uma característica comum das respostas comportamentais de bovinos, tanto em situações estressantes físicas, quanto psicológicas. Medir as respostas vocais, durante o manuseio de procedimentos desagradáveis, como um indicador de bem-estar animal tem uma vantagem, em comparação com medições fisiológicas, por ser totalmente nãoinvasiva. (Watts et al., 2001).

Yeon et al. (2006) após os resultados de sua pesquisa, sugeriram que a análise de vocalizações pode ser um indicador de estado fisiológico e emocional das vacas. Com isso, os mesmos autores abordam que estudos sobre a vocalização dos animais como meio de compreensão de seu estado comportamental devem ser incentivados pelos órgãos fomentadores de estudos nesta área.

Uma das grandes implicações em pesquisas para avaliação de respostas comportamentais é a definição das atividades, pois as mesmas podem variar entre espécies, categorias, sistemas produtivos, entre outros.

Faerevik et al. (2006) avaliaram as respostas comportamentais de bezerros leiteiros com seis semanas de idade sobre diferentes formas de isolamento (isolamento individual, isolamento com outro bezerro conhecido e isolamento com outro bezerro desconhecido), em uma baia desconhecida por 20 minutos, conforme a tabela I. Os autores observaram que os bezerros responderam a separação do grupo, com aumento de vocalização (tabela II). No entanto, nenhum bezerro vocalizou na presença de um bezerro familiar. Além disso, a separação, sem um companheiro animal (isolamento) levou maior tempo de imobilidade, menos exploração da baia e menor atividade locomotora (passos). Com esses resultados os autores verificaram que há efeito de familiaridade do animal companheiro sobre as vocalizações, concluindose que a presença de um bezerro familiar parece ter um efeito calmante (bezerros não vocalizando) em relação à separação com um bezerro desconhecido. Além de um efeito 
Tabela II. Resultados do teste de isolamento (médias \pm desvio padrão). (Results of the isolation test (mean standard deviation)).

\begin{tabular}{|c|c|c|c|c|}
\hline \multirow[t]{2}{*}{ Comportamento } & \multicolumn{3}{|c|}{ Estímulo } & \multirow[t]{2}{*}{$\mathrm{p}$} \\
\hline & Conhecido & Desconhecido & Sozinho & \\
\hline Bezerros vocalizando $\left(n^{\circ}\right)$ & $0^{a}$ & $5^{b}$ & $8^{\mathrm{b}}$ & $<0,05$ \\
\hline Frequência cardíaca (batimentos/min.) & $128,27 \pm 2,61^{\mathrm{a}}$ & $119,11 \pm 2,72^{\mathrm{a}}$ & $63,83 \pm 6,52^{b}$ & $<0,01$ \\
\hline Passos $\left(n^{0}\right)$ & $95,58 \pm 4,90^{\mathrm{a}}$ & $86,67 \pm 5,57^{a}$ & $63,83 \pm 6,52^{b}$ & $<0,001$ \\
\hline Imobilidade (min.) & $2,93 \pm 0,05^{a}$ & $2,81 \pm 0,54^{a}$ & $10,02 \pm 0,90^{b}$ & $<0,001$ \\
\hline Exploração (min.) & $15,85 \pm 0,52^{\mathrm{a}}$ & $16,08 \pm 0,50^{a}$ & $9,74 \pm 0,86^{a}$ & $<0,001$ \\
\hline Contato social (min.) & $0,66 \pm 0,12^{\mathrm{a}}$ & $0,46 \pm 0,06^{a}$ & - & n.s. \\
\hline
\end{tabular}

Médias com letras diferentes na linha, diferem entre si $(p<0,05)$.

Fonte: Faerevik et al. (2006).

calmante de um bezerro familiar, que também era esperado, o tempo de contato social quando os animais foram separados com um bezerro familiar deveria ser maior do que quando separados com um bezerro estranho, mas não foi isso que ocorreu.

Em alguns países, já existem legislações que regulamentam a criação de bezerros. Seguindo a legislação sueca, um bezerro é definido pelo animal com idade de até seis meses de vida, esta definição é também utilizado pela legislação da União Européia, com isso não é permitido amarrar os bezerros (DFS, 2007:5), sendo o mesmo alocado em baia especial, com aquecimento estável (tabela III). Baias para bezerros com menos de um mês de vida devem ter o piso coberto com palha ou outro material equivalente (SFS, 2007:1395). As baias individuais devem

Tabela III. Espaço mínimo para bezerros em baias individuais (DFS, 2007:5). (Minimum space for calves in individual pens).

\begin{tabular}{cccc}
\hline PM & C & L & A \\
\hline 60 & 1,20 & 1,00 & 1,20 \\
90 & 1,40 & 1,00 & 1,40 \\
\hline
\end{tabular}

PM: Peso máximo (kg); C: Comprimento (m); L: Largura $(\mathrm{m})$; A: Área $\left(\mathrm{m}^{2}\right)$ ter paredes fechadas até $0,80 \mathrm{~m}$ e o restante da parede aberta para que ocorra contato visual e proximidades da cabeça entre bezerros (DFS, 2007:5).

Roussel et al. (2006) avaliaram em ovelhas o efeito da separação da fêmea por alguns minutos por dia, duas vezes por semana, na fase pré-parto sobre a resposta ao isolamento da matriz após o parto, com os seguintes tratamentos: isolamento (ISO, $\mathrm{n}=$ 20 fêmeas) e nenhuma perturbação (CON, $\mathrm{n}=20$ fêmeas), em que os isolamentos foram visuais, pois a ovelha era retirada do grupo por um funcionário manejador. Os autores verificaram que as ovelhas isoladas estavam mais motivadas para permanecer em contato com seus cordeiros na presença de um ser humano. Os autores abordam também que houve uma tendência de elevação para a resposta do cortisol nas ovelhas grávidas durante o tratamento (estresse).

Embora o isolamento seja conhecido por ser um estressor psicológico importante em ovinos, Roussel et al. (2006), mostraram evidências de habituação ao procedimento de isolamento em ovelhas. Aprofundando um pouco mais, Roussel-Huchette et al. (2008) pesquisaram se existe uma transmissão desse estresse do isolamento da fêmea para a prole. Avaliando ovelhas, os autores isolaram 10 vezes, durante as últi- 
Tabela IV. Etograma do comportamento verificadas durante o pré-teste e pós-tratamento. (Ethogram of the behavior observed during the pre-test and post-treatment).

\begin{tabular}{ll} 
Variação comportamental & Definição \\
\hline Nenhuma & A perna traseira não era movida dentro dos limites temporais do teste. \\
Emmovimento & O casco da perna traseira é movido na horizontal, mas o casco não se \\
afasta do piso. & O casco da perna traseira é levantado do piso de uma forma calma. \\
Elevada & O casco da perna traseira é batido no piso ou movimentado em alta \\
Escoicear & velocidade (coice).
\end{tabular}

Fonte: Herskin et al. (2007).

mas seis semanas de gestação, verificando o isolamento (ISOL, $\mathrm{n}=20$ ovelhas), e nenhuma perturbação (CON, $n=20$ ovelhas). O peso corporal dos cordeiros dos animais isolados foi superior ao controle, e a concentração de cortisol, aos 30 e 90 dias de idade, não diferiu entre os animais isolados e o controle.

As correlações entre os escores de comportamento dos cordeiros e os fisiológicos das ovelhas foram significativas em estudo de Roussel-Huchette et al. (2008). Em comparação com cordeiros nascidos de ovelhas sem isolamento, um menor efeito do estresse pré-natal foi encontrado na reatividade emocional dos cordeiros nascidos de ovelhas isoladas durante a gestação. Estas diferenças podem ser explicadas por diferentes hipóteses: em primeiro lugar, ovelhas isoladas habituaram rapidamente ao tratamento do estresse, repassando essa habituação através da placenta ao feto, o que poderia ter modificado o desenvolvimento do cérebro em comparação com os fetos de ovelhas sem isolamento (Roussel et al., 2006). A segunda hipótese seria de que as ovelhas repassam essa habituação a prole nos primeiros dias após seu nascimento.

Roussel et al. (2005) verificando os mesmos tratamentos, só que em cabras, tentaram justificar esse fenômeno, separando os cabritos de suas mães após consumir o colostro, e observaram melhores resultados dos animais que suas mães foram isoladas do que as controle. Sendo assim a modificação da reatividade emocional do cabrito poderia ser em parte uma consequência da quantidade de cortisol que atravessam a barreira placentária e modificar o desenvolvimento cerebral do feto. No entanto, é difícil comparar os estudos comportamentais existentes por causa da variedade de espécies e processos utilizados para estudo de estresse pré-natal.

Herskin et al. (2007) trabalharam com vacas leiteiras testando os tratamentos: controle (con), isolamento espacial (res, animais contidos com cabrestos), isolamento visual (isol, animais isolados em baias), conforme descrição das atividades (tabela IV). Os autores verificaram que no início (até 10 minutos) da indução dos tratamentos, os níveis de cortisol foram semelhantes, entretanto até o tempo intermediário da avaliação (aproximadamente de 35 minutos), os níveis foram variando diferindo entre os tratamentos, sendo que o isolamento visual apresentou uma maior diferença dos demais, seguido pela restrição e controle, respectivamente (figura 1). No final da avaliação (aos 70 minutos) os níveis de cortisol se assemelharam novamente, tendenciando também a uma habituação do isolamento.

Portanto nas respostas comportamentais 




Figura 1. Concentração plasmática de cortisol do tratamento controle (CON), isolamento espacial (RES, animais contidos com cabrestos), isolamento visual (ISOL, animais isolados em baias) ao longo do tempo de avaliação. Fonte: Herskin et al. (2007). (Concentration plasma of cortisol of the control treatment (CON), spatial isolation (RES, halter with animals contained), visual isolation (ISOL, single animals in pens) along the time of evaluation).

da pesquisa de Herskin et al. (2007), os resultados encontrados afirmaram uma menor movimentação em alta velocidade (escoicear), o que provavelmente ocorreu devido a limitação espacial, que foi induzido tanto no tratamento restrição onde o animal era contido com cabresto, quanto no tratamento isolamento onde o animal foi contido em baia (tabela V).

Alguns autores na literatura que ava-

Tabela V. Respostas comportamentais do controle, restrição e isolamento. (Behavioral responses of control, restraint and isolation).

\begin{tabular}{lcccccr}
\hline \multirow{2}{*}{ Tipo de resposta (\%) } & \multicolumn{2}{c}{ Controle } & \multicolumn{2}{c}{ Restrição } & \multicolumn{2}{c}{ Isolamento } \\
& Antes & Depois & Antes & Depois & Antes & Depois \\
\hline Nenhuma & 27,3 & 9,1 & 22,2 & 18,5 & 27,6 & 21,2 \\
Em movimento & 18,2 & 27,3 & 18,5 & 20,4 & 18,2 & 37,9 \\
Elevada & 28,8 & 34,8 & 22,2 & 37,0 & 23,9 & 21,2 \\
Escoicear & 25,8 & $28,8^{\text {a }}$ & 37,0 & $24,1^{\text {b }}$ & 30,3 & $19,7^{\text {c }}$
\end{tabular}

Fonte: Herskin et al. (2007).

Archivos de zootecnia vol. 60 (R), p. 60. 
Tabela VI. Respostas aos tratamentos (média \pm desvio-padrão). (Responses to treatments (mean \pm standard deviation)).

\begin{tabular}{lcc}
\hline Tratamento & $\begin{array}{c}\text { Batimentos } \\
\text { por minuto }\end{array}$ & $\begin{array}{c}\text { Número de } \\
\text { movimentos }\end{array}$ \\
\hline Com espelho & $91,5 \pm 1,4^{\text {b }}$ & $32,9 \pm 3,5$ \\
Sem espelho & $98,5 \pm 1,3^{\text {a }}$ & $42,3 \pm 3,9$ \\
\hline
\end{tabular}

${ }^{a b}$ diferenças significativas na $p<0,05$.

Fonte: Piller et al. (1999).

liaram os aspectos comportamentais de animais, concluíram que pode ocorrer um efeito de indivíduo (genético) nas respostas de estudos comportamentais, com isso, Watts et al. (2001) avaliaram, em 130 bezerros de corte, o efeito das diferenças dos pais, raça, sexo, idade e peso, nas respostas vocais dos animais ao isolamento visual. Foi verificado efeito da idade, peso, touro e vaca para todas as variáveis de respostas vocais (frequência, intensidade, duração, entre outras) submetidas ao isolamento. Já para sexo, não foi encontrada diferenças significativas entre as respostas avaliadas.

Watts et al. (2001) afirmaram que não é possível saber se o touro exerce maior influência genética que as vacas sobre as características vocais da prole. No entanto, é certo que uma proporção do comportamento vocal de um bezerro, é herdada dos pais, independente das condições de criação e de aprendizagem.

Piller et al. (1999) estudaram o efeito da reflexão da própria imagem do animal através

\section{BIBLIOGRAFIA}

Eriksson, I. 2009. Optimal gruppstorlek for kalvar som utfodras i transponderstyrda kalvammor. Swedish University of Agricultural Sciences Uppsala. Uppsala. 64 pp.

Esteves, I., Andersen, I-L. and Naevdal, E. 2007. Group size, density and social dynamics in farm animals. Appl. Anim. Behav. Sci., 103: 185-204. Faerevik, G., Jensen, M.B. and Boe, K.E. 2006. de espelhos sobre o isolamento visual, com o intuito de minimizar o estresse.

Os resultados indicaram que o espelho diminuiu os batimentos por minuto, e não provocou alterações no número de movimentos. Portanto, o mesmo salienta que não há evidência que indique que os animais estão cientes de que suas reflexões são imagens próprias, como também não se sabe se eles encaram a sua reflexão como uma representação razoável da mesma espécie, pois a imagem espelhada é desprovida de sentidos olfativos, gustativos, vocal e sinais em relevo (tabela VI).

\section{CONSIDERAÇÕESFINAIS}

Em animais, o isolamento visual e/ou espacial altera as respostas comportamentais e produtivas. O isolamento visual possui uma tendência a apresentar maiores alterações no animal que o espacial.

A repetição do isolamento por consecutivas vezes provoca uma habituação do indivíduo ao mesmo, acarretando em diminuição ou até nulidade da alteração comportamental.

Para avaliações de bem-estar dos animais, recomenda-se a mensuração através das reações comportamentais, por ser um método não-invasivo.

Recomendam-se mais pesquisas nesta área tendo em vista a sua importância para os sistemas de produção animal, principalmente com a verticalização do mesmo, onde atenua as reações e variações de respostas do indivíduo.

Dairy calves social preferences and the significance of a companion animal during separation from the group. Appl. Anim. Behav. Sci., 99: 205-221.

Fredriksson, M., Ventorp, M. and Herlin, A. 2006. Optimal valfard och halsa for kalvar. SLU Alnarp, Institutionen för jordbrukets biosystem och teknologi. Lantbrukets byggnadsteknik. 20 pp. 
Herskin, M.S., Munksgaard, L. and Andersen, J.B. 2007. Effects of social isolation and restraint on adrenocortical responses and hypoalgesia in loose-housed dairy cows. J. Anim. Sci., 85: 240-247.

Kilgour, R.J., Melville, G.J. and Greenwood, P.L. 2006. Individual differences in the reaction of beef cattle to situations involving social isolation, close proximity of humans, restraint and novelty. Appl. Anim. Behav. Sci., 99: 21-40.

Kondo, S. and Hurnik, J.F. 1988. Behavioural and physiological responses to spatial novelty in dairy cows. J. Anim. Sci., 68: 339-343.

Mckittrick, C.R., Blanchard, D.C. and Hardy, M.P. 2009. Social stress effects on hormones, brain, and behavior. Horm. Behav., 1: 333-365.

Piller, C.A.K., Stookey, J.M. and Watts, J.M. 1999. Effects of mirror-image exposure on heart rate and movement of isolated heifers. Appl. Anim. Behav. Sci., 63: 93-102.

Roussel, S., Boissy, A. and Montigny, D. 2005. Gender-specific effects of prenatal stress on emotional reactivity and stress physiology of goat kids. Hormones and Behavior, 47: 256-266.

Roussel, S., Hemswortha, P.H., Lerustea, H., Whitea, C., Duvaux-Ponterb, C., Nowakc, R. and Boissyd, A. 2006. Repeated transport and isolation during pregnancy in ewes: Effects on the reactivity to humans and to their offspring after lambing. Appl. Anim. Behav. Sci., 97: 172-189.
Roussel-Huchette, S., Hemsworth, P.H., Boissy, A. and Duvaux-Ponter, C. 2008. Repeated transport and isolation during pregnancy in ewes: Differential effects on emotional reactivity and weight of their offspring. Appl. Anim. Behav. Sci., 109: 275-291.

Watts, J.M., Stookey, J.M., Schmutz, S.M. and Waltz, C.S. 2001. Variability in vocal and behavioural responses to visual isolation between full-sibling families of beef calves. Appl. Anim. Behav. Sci., 70: 255-273.

Yeon, S.C., Jeon, J.H., Houpt, K.A., Chang, H.H., Lee, H.C. and Lee, H.J. 2006. Acoustic features of vocalizations of Korean native cows (Bos taurus coreanea) in two different conditions. Appl. Anim. Behav. Sci., 101: 1-9.

\section{LEGISLAÇÕES}

EU legislation Council directive 97/2/EEC.

EU council's recommendation concerning cattle, adopted by the Standing Committee. Adopted by the Standing Committee on 21 October 1988. Disponível em <http://www.coe.int/t/e/legal_ affairs/legal_cooperation/biological_safety, _use_of_animals/Farming/Rec\%20cattle $\%$ 20E.asp>.

DFS, 2007:5. Djurskyddsmyndighetens foreskrifter och allmanna rad om djurhallning inom lantbruket, saknr L100. Skara.

SFS, 2007:1395. Djurskyddsforordningen. Jordbruksdepartementet. 